\title{
A Lower Bound on Snap-Through Instability of Curved Beams under Thermomechanical Loads
}

\author{
Ilinca Stanciulescu ${ }^{\mathrm{a}, *}$, Toby Mitchell ${ }^{\mathrm{b}}$, Yenny Chandra ${ }^{\mathrm{a}}$, Thomas Eason ${ }^{\mathrm{c}}$, \\ Michael Spottswood ${ }^{\mathrm{C}}$ \\ ${ }^{a}$ Rice University, Department of Civil and Environmental Engineering, 6100 Main \\ Street, Houston, TX, 77005, U.S.A \\ ${ }^{b}$ University of California at Berkeley, Department of Civil Engineering, Berkeley, CA, \\ 94720, U.S.A \\ ${ }^{c}$ Air Force Research Laboratory, Structural Sciences Center, 2790 D. Street, WPAFB, \\ OH, U.S.A.
}

\begin{abstract}
A nonlinear finite element formulation (three dimensional continuum elements) is implemented and used for modeling dynamic snap-through in beams with initial curvature. We identify a nontrivial (nonflat) configuration of the beam at a critical temperature value below which the beam will no longer experience snap-through under any magnitude of applied quasistatic loadfor beams with various curvatures. The critical temperature is shown to successfully eliminate snap-through in dynamic simulations at quasistatic loading rates. Thermomechanical coupling is included in order to model a physically minimal amount of damping in the system, and the resulting post-snap vibrations are shown to be thermoelastically damped. We propose a test to determine the critical snap-free temperature for members
\end{abstract}

\footnotetext{
*Corresponding author. Tel.: +1713 348 4704; fax: +1713 3485268 .

Email address: ilinca@rice.edu (Ilinca Stanciulescu)
} 
of general geometry and loading pattern; the analogy between mechanical prestress and thermal strain that holds between the static and dynamic simulations is used to suggest a simple method for reducing the vulnerability of thin-walled structural members to dynamic snap-through in members of large initial curvature via the introduction of initial pretension.

Keywords: snap-through, finite element, curved beam, solid (continuum) elements, thermomechanical loads

\section{Introduction}

Thin structural members can dynamically jump between multiple equilibrium configurations when subjected to mechanical forces, acoustic vibrations, and thermal loads like those encountered by aerospace vehicles in extreme operating conditions. This process, commonly referred to as snap-through, can cause large-amplitude structural vibrations, induce fatigue, and lead to

global instability. Such vibrations can have a chaotic pattern and therefore can be difficult to control [1]. Avoiding snap-through is therefore highly desirable in the design of thin-walled structures such as aircraft and spacecraft.

In this paper, the existence of a critical temperature below which an initially curved beam will no longer experience snap-through at any applied quasi-static load level is demonstrated. This temperature provides a lower bound below which snap-through instability no longer occurs. This temperature also corresponds to a nontrivial (that is, nonflat) deformed beam configuration, a fact that is not apparent through methods used to characterize snap-through that are limited to modeling small deflections. We argue that such a limit can be obtained for a variety of static loads and that it also 
reduces the amplitude of the oscillations when loads are applied at dynamic rates. Through numerical experiments we show this limit to be found at states characterized by specific properties associated with the energy of the system and the directional derivatives of this energy function.

Experimental characterizations of snap-through under mechanical vibrations and combined thermal and mechanical loads have existed for several decades $[2,3,4]$, but numerical modeling of snap-through remains an area of active research. In the general case, snap-through involves combined flexural, shear, and normal stresses, large time varying deflections and rotations, and thermal effects. Nonlinear coupling between these effects makes snapthrough challenging to model accurately. An extensive literature exists on the subject, with models describing different degrees of geometric nonlinearity $[5,6]$, material nonlinearity $[7,8,9]$, thermal and acoustic loading $[10,11,12]$, and various combinations of these factors [13, 14].

Previous research on snap-through falls into three general categories: (1) limited-deflection models (based on the von Karmann or Duffing equations for plates), (2) elastica models (based on specialized analytical techniques going back to Euler), and (3) nonlinear (finite-deformation) finite element models.

The first category suffers from the most significant limitation: these models can only describe member deflections of less than 2.5 times the member thickness [5]. This is especially problematic because large initial curvatures that may be present by design, e.g., in a rocket booster hull or aircraft wing, will lead to larger snap-through loads and therefore more severe and damaging post-snap vibrations, none of which can be modeled with limited 
deflection formulations.

Elastica methods are valid at a theoretically unlimited range of deflections and initial curvatures, at the cost of increased mathematical complexity. These techniques have only recently been extended to the solution of thermal and snap-through problems $[15,16,17]$. This is most likely due to the increased ease of solving the equations numerically, e.g., by shooting methods $[18,19]$. Although there is no apparent limitation to obtaining the results discussed in this paper by elastica methods (other than possible limitations to particular instances of the theory, e.g., the common omission of shear strain), they are not as well-developed for general classes of problems as nonlinear finite element methods.

Nonlinear finite element methods are the most general of the methods available to model snap-through, an advantage for which they sacrifice the direct, problem-specific analytical insight that is available via the first two methods. This lack of transparency is more than compensated by the versatility to model geometric nonlinearity, thermomechanical coupling, large strains, material nonlinearity, fluid-structure interaction in aircraft, etc.

The nonlinear finite element method is chosen for this study; it is capable of including the full range of nonlinearities that influence snap-through and can easily model large deflections. Beam and shell models, usually the most computationally efficient choice for finite element modeling of thin structural members, always involve some simplifications of the underlying threedimensional kinematics that can lead to artificial stiffness under particular load states (locking). These kinematic assumptions that are built into the formulation of structural elements can lead to inaccurate solutions [20]. In 
this work we avoid such issues as well as the locking sometimes present when linear elements are used by choosing to work with three-dimensional (solid) quadratic elements only. In this paper, the numerical simulations are performed with the Finite Element Analysis Program (FEAP), an open source research code, which provides a framework for finite element simulations where we can formulate and implement additional elements, constitutive models and solution schemes via user subroutines. The formulations utilized for the analysis discussed in this paper are a mix of FEAP original elements and user routines. [21].

By thermomechanical coupling we refer only to the coupling between elastic deformation and thermal effects via the thermal strain terms in the equations of mechanical equilibrium and the structural elastic heating term in the heat equation. Although thermoelastic coupling is present in all materials, it is often neglected; however, for large-amplitude vibrations, the thermal gradient induced between the compressive and tensile fibers of a vibrating member due to purely elastic deformation results in significant heat conduction and therefore loss of energy via thermoelastic damping. We do not include any other mechanisms of dissipation besides thermoelastic damping, so the damping in our study is physically minimal, and the resulting vibrations are exaggerated relative to real physical systems.

In many studies $[22,23]$, thermal strain due to applied temperature is included without modeling thermoelastic coupling: temperature changes are thus treated as a simple mechanical expansion or contraction of the material. This can be useful in tracing static solution paths, and may also give information about the temperature-sensitivity of a particular structure, but 
the absence of thermoelastic coupling can impact the accuracy of dynamic simulations, especially as amplitudes of vibration become large. We employ the strain-only method in our static simulations, but move to full coupling in order to capture relevant dynamic coupling effects.

Modeling of thermomechanical coupling also requires the selection of an appropriate thermomechanical material constitutive law. A hyperelastic constitutive law based on a modified neo-Hookean material that is appropriate for metals has been proposed in previous studies [24]. Since we will be primarily concerned with metals, the modified thermomechanical neo-Hookean material law is appropriate and will be valid at a much larger range of strains than the thermomechanical St. Venant-Kirchhoff law.

Failure to account for any of the above modeling concerns can significantly impact the accuracy of dynamic simulation results. The formulation adopted for this study accounts for the geometric nonlinearity, large strains, and thermomechanical coupling effects required to describe snap-through. It is a nonlinear finite element formulation, with quadratic hexahedral (or tetrahedral) elements that uses the adiabatic staggered scheme and the constitutive law detailed in [24].

The initial boundary value problem is formulated as follows: For all $t \in \mathbb{I}$ find the motion $(\phi)$ and temperature $(T)$ fields such that

$$
\begin{aligned}
\rho_{0} \frac{\partial^{2}}{\partial t^{2}} \boldsymbol{\phi} & =\operatorname{div} \boldsymbol{P}+\boldsymbol{b} \\
c \dot{T} & =\mathcal{D}-\mathcal{K}-J \operatorname{div}[\boldsymbol{q} / J]+\mathcal{R}
\end{aligned}
$$

with boundary conditions $\boldsymbol{P} \boldsymbol{n}_{0}=\overline{\boldsymbol{t}}$ on $\Gamma_{\sigma} \times \mathbb{I}, \boldsymbol{\phi}=\overline{\boldsymbol{\phi}}$ on $\Gamma_{\phi} \times \mathbb{I}, \boldsymbol{q} \boldsymbol{n}_{0}=\bar{q}$ on $\Gamma_{q} \times \mathbb{I}, T=\bar{T}$ on $\Gamma_{q} \times \mathbb{I}$ and initial conditions $\left.\boldsymbol{\phi}\right|_{t=0}=\boldsymbol{I}$ in $\bar{\Omega},\left.\frac{\partial}{\partial t} \boldsymbol{\phi}\right|_{t=0}=\boldsymbol{V}_{0}$ 
in $\bar{\Omega},\left.T\right|_{t=0}=T_{0}$ in $\bar{\Omega} . \Omega \subset \mathbb{R}^{n_{d}}$ is the domain in the reference configuration, $n_{d}$ is the number of spatial dimensions in the problem, $\boldsymbol{P}$ is the first Piola Kirchhoff stress tensor, $\boldsymbol{f}$ is the prescribed body force, $c$ is the heat capacity, $\mathcal{D}$ is the mechanical dissipation, $\mathcal{K}$ the heating from the Joule effect [25], $\mathcal{R}$ the prescribed heat source term, and $J$ the Jacobian of the deformation. denotes the prescribed value of the quantity $\bullet$ over the appropriate boundary region or at the initial time. The generic problem described here can be completed with a constitutive law prevailing in the body and the Fourier law for heat conduction is assumed to relate the local heat flux $\boldsymbol{q}$ to the temperature gradient, $\boldsymbol{q}=-\left[\begin{array}{ll}k & \mathbf{1}\end{array}\right] \nabla T$, where $k$ is the thermal conductivity and $\mathbf{1}$ is the identity tensor. For the purpose of this paper, the constitutive law is assumed to have temperature-dependent constitutive moduli. After a spatial discretization is applied (e.g., finite element) the system (1) can be expressed as a system of ordinary differential equations with two coupled partitions: mechanical (second order) and thermal (first order).

For the transient solution we use the adiabatic staggered scheme for coupled thermoelastic boundary value problems developed in [24]; this approach splits the solution into partitions such that the dissipative property of the original problem is maintained in the partitioned problem. The scheme consists of (1) a standard finite deformation mechanical phase, formulated such that it is solved at constant entropy, and (2) a heat conduction phase, formulated such that it is solved at fixed deformation.

We also adopted the constitutive law from [24], p. 760, eqs. $82-83$ for a for a regularized compressible neo-Hookean material. This is a law that performs well for metals and is given by the thermomechanical strain energy 
density function

$$
\Psi(\mathbf{C}, \Theta)=W(\overline{\mathbf{C}})+U(J)+M(J, \Theta)+T(\Theta)
$$

where $W, U, M$, and $T$ are the mechanical deviatoric, mechanical volumetric, thermomechanical coupling, and thermal-only terms respectively, given by

$$
\begin{aligned}
W(\overline{\mathbf{C}}) & =\frac{1}{2} \mu(\operatorname{tr}(\overline{\mathbf{C}})-3)=\frac{1}{2} \mu\left(J^{-\frac{2}{3}} \operatorname{tr}(\mathbf{C})-3\right) \\
U(J) & =\frac{1}{2} K(\ln J)^{2} \\
M(J, \Theta) & =-3 K \alpha\left(\Theta-\Theta_{r e f}\right) \ln J \\
T(\Theta) & =\rho c_{m}\left[\left(\Theta-\Theta_{r e f}\right)-\Theta \ln \left(\frac{\Theta}{\Theta_{r e f}}\right)\right] .
\end{aligned}
$$

where $\mathbf{C}$ is the right Cauchy-Green tensor, $\mu$ is the shear modulus, $\rho c_{m}$ is the heat capacity, $\Theta_{\text {ref }}$ is the reference temperature, $K$ is the bulk modulus, $\alpha$ is the coefficient of thermal expansion and $J$ is the Jacobian of deformation.

For the detailed derivation of the components of the finite element formulations utilized in this work, the interested reader is refered to [26].

The rest of the paper is organized as follows. In section 2 we describe the geometry of the system under consideration and briefly describe the solution methods utilized to retrieve equilibrium paths. Section 3 is dedicated to numerical experiments that trace such equilibrium paths. In this section, we identify special equilibrium configurations corresponding to boundaries in the parameter space that separate domains with different stability behaviors. Among these, the quasi-static temperature limit that we will refer to as monotonic, i.e., that value of the temperature below which the equilib- 


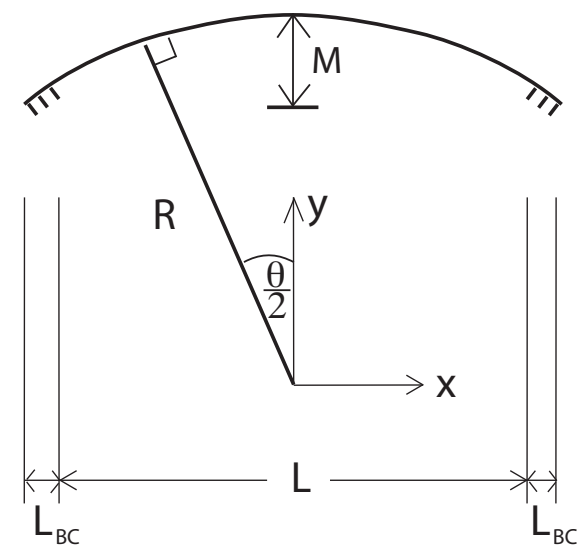

Figure 1: Geometry for semicircular beam.

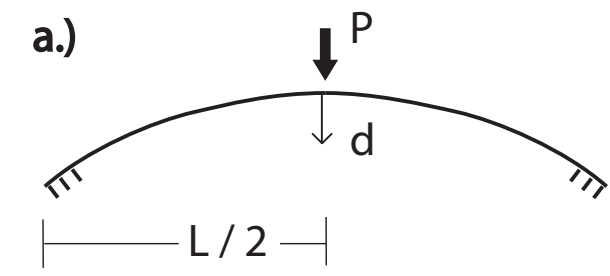

b.)

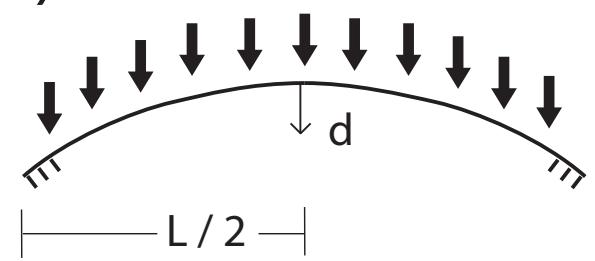

Figure 2: Concentrated (a) and distributed (b) loads. The point load is the primary case considered.

rium path is a monotonic curve, and snap-through is no longer encountered. Section 4 demonstrates, through a variety of transient simulations, that the monotonic temperature also identifies a limit below which the amplitude of the dynamic oscillations is drastically reduced and asymptotically reaches 0 at temperatures in the neighborhood of the quasi-static limit. Section 5 provides a generalization of the concept of monotonic temperature and shows that information regarding such stability limits is no longer available when some specific finite element formulations (e.g., the Timoshenko beam) are utilized. When kinematic approximations such as those used by the Timoshenko beam are used, information available in the higher derivatives of the energy is lost in such formulations. 


\section{Static Analysis of a Curved Beam}

A planar curved beam described by an arc of a circle is chosen as the test problem to explore the effect of applied temperature and initial beam curvature on snap-through. The problem is solved in two phases (mechanical and thermal.) The beam geometry is completely specified by the length of the horizontal projection between the end points $L$, the projection of the lengths of the supports $L_{B C}$, and the radius of curvature of the beam $R$ (Figure 1). The primary load case considered is a point load $P$ in the negative y-direction located at the midpoint between the supports (Figure 2a). A distributed load $p$, applied as a uniform force in the negative $\mathrm{y}$-direction on each node of the finite element mesh, is also considered (Figure 2b).

In simulations, eight different beams were utilized, with $R$ varying from $762 \mathrm{~mm}$ (30 in.) to $5080 \mathrm{~mm}$ (200 in.) The effect of temperature variation on the load-deflection behavior in the case of larger curvatures was found to be very small, so such test problems were abandoned.

Table 1 summarizes the geometry of the beams: $M$ is the arch rise, $\kappa=1 / R$ is the curvature of the beam, $\theta$ is the angle subtended by the

\begin{tabular}{rrrlll}
\hline Beam & $\mathrm{R}[\mathrm{mm}]$ & $\mathrm{M}[\mathrm{mm}]$ & $\kappa[1 / \mathrm{mm}]$ & $\theta / 2[\mathrm{rad}]$ & $\mathrm{M} / \mathrm{h}$ \\
\hline 1 & 762.0 & 21.84 & $1.312 \cdot 10^{-3}$ & 0.2400 & 86.0 \\
2 & 1270.0 & 12.99 & $0.787 \cdot 10^{-3}$ & 0.1431 & 51.1 \\
3 & 1828.8 & 8.98 & $0.546 \cdot 10^{-3}$ & 0.0990 & 35.3 \\
4 & 2133.6 & 7.70 & $0.469 \cdot 10^{-3}$ & 0.0850 & 30.3 \\
5 & 2438.4 & 6.74 & $0.410 \cdot 10^{-3}$ & 0.0744 & 26.5 \\
6 & 3048.0 & 5.39 & $0.328 \cdot 10^{-3}$ & 0.0595 & 21.2 \\
7 & 3810.0 & 4.31 & $0.262 \cdot 10^{-3}$ & 0.0475 & 17.0 \\
8 & 5080.0 & 3.23 & $0.169 \cdot 10^{-3}$ & 0.0357 & 12.7 \\
\hline
\end{tabular}

Table 1: Geometry of Curved Beams. 


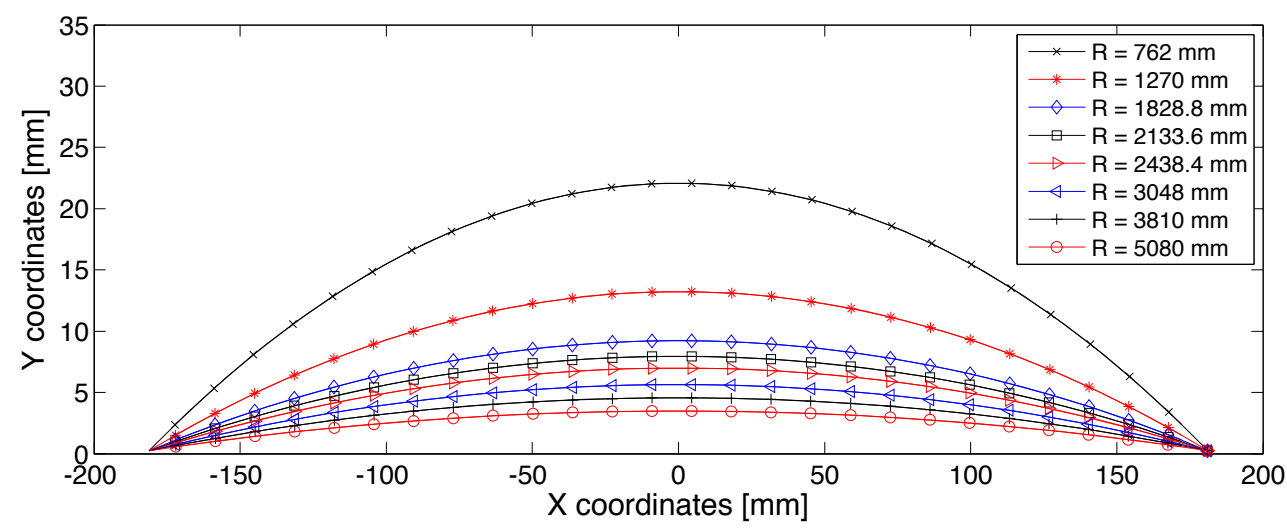

Figure 3: Initial beam configurations.

beam, and $M / h$ is the ratio of $M$ to the thickness $h$ of the beam. Figure 3 shows the different initial beam curvatures. For all beams, the projection length is $L=304.8 \mathrm{~mm}$ (12 in.), the thickness $h=0.508 \mathrm{~mm}$ (0.02 in) and the transverse depth $b=12.7 \mathrm{~mm}$ ( $0.5 \mathrm{in}$.). The ends of the beam are held fixed over a length $L_{B C}=28.7 \mathrm{~mm}$ (1.125 in.) on each end. Material properties are those of steel, given in Table 2.

\begin{tabular}{llll}
\hline Property & Symbol & Value & Units [N-mm-s-K] \\
\hline Young's modulus & $E$ & 206483 & $\mathrm{~N} / \mathrm{mm}^{2}$ \\
Poisson's ratio & $\nu$ & 0.28 & - \\
Density & $\rho$ & $7.834 \times 10^{-9}$ & $\mathrm{~N} \mathrm{~s}^{2} / \mathrm{mm}^{4}$ \\
Conductivity & $k$ & 45.0 & $\mathrm{~N} / \mathrm{s} \mathrm{K}$ \\
Specific heat & $c_{m}$ & $434 \times 10^{6}$ & $\mathrm{~mm}^{2} / \mathrm{s}^{2} \mathrm{~K}$ \\
Thermal expansion & $\alpha$ & $14 \times 10^{-6}$ & $\mathrm{~mm} / \mathrm{mm} \mathrm{K}$ \\
\hline
\end{tabular}

Table 2: Material properties of thermoelastic beam.

In this paper we concentrate our attention to a system that is effectively two-dimensional; nevertheles, we use a finite element model with full threedimensional capabilities. Mesh refinement studies were performed and the level of refinement adequate to accurately capture the snap-through loads 
lead to a model with approvimately 20,000 degrees of freedom. Note that linear elements suffer from shear locking and are not appropriate for snapthrough problems, or for any problems involving large deformation. This problem is avoided by using quadratic elements.

The formulation adopted [24] treats the coupling through an adiabatic staggered approach. For the quasistatic simulations of systems under constant temperature presented in the next section, the mechanical phase is solved in isolation from the thermal phase, treating the applied temperature change as a conservative, purely mechanical expansion or contraction of the beam material. Many other studies have employed this method, but, with a few exceptions $[15,16,6]$, they have been limited to beams of small initial curvature.

The case of the point load along the line of symmetry of the beam provides an easy interpretation of the simulation results. If the symmetry is not broken by a bifurcation, as may occur if the second, asymmetric buckling mode emerges [27], the plots of midpoint deflection $d$ vs. load value $P$ will indicate loss of stability and locate the snap-through and snap-back loads at the points where $\partial P / \partial d=0$. The ability to obtain such information is completely dependent on the symmetry of the problem: in cases without a clear line of symmetry it is no longer possible to obtain information about the snapthrough point from the load-deflection curve, and even in the symmetric case of the distributed load this breaks down at heightened temperatures. The simplicity of the symmetric concentrated load case will make it easier to develop insight into the stability behavior of the beam, which we will then need to generalize to cases where important behaviors are no longer apparent 
from the load-deflection curves.

\subsection{Static Solution Paths}

Snap is inherently a dynamic phenomenon; since the static equilibrium path is interrupted by an unstable region, as the load is increased, the system must dynamically jump past the unstable region and onto a stable region capable of bearing loads above the snap-through load. Unlike column buckling, there is no stable branch that the system can follow continuously along the equilibrium path. Nevertheless, useful information can be obtained by studying the static solution paths. ${ }^{1}$ The lack of inertia allows us to simplify the analysis, since there is no need to consider the effects of varying loading rates. Moreover, the unstable equilibrium path between the stable regions discloses information that is relevant to the dynamic case. We mapped the static equilibrium path by using an pseudo arc-length procedure to traverse the unstable region.

The nonlinear continuum finite element method recovers information that is unavailable from methods based on the beam or plate (restricted kinematics) equations. In particular, there exists a nontrivial deformed configuration, at a temperature below the zero-stress reference temperature $\Theta_{\text {ref }}$, that will not experience snap-through at any applied static load value. The value of this temperature depends on the initial beam geometry, the boundary conditions, and the pattern of applied load. Simulations suggest that any beam that is cooled below this temperature no longer experiences snap-through.

\footnotetext{
1 "Static" refers to the mode of recovery of information on snap-through, not an actual physical scenario.
} 
The subsequent sections in this paper are concerned with developing tests to establish this lower bound on thermoelastic instability and determining whether this limit, which is derived from purely mechanical considerations, is sufficient to eliminate dynamic snap-through in beam simulations that incorporate full thermomechanical coupling.

\section{Numerical Experiments. The Quasi-static Analysis}

The zero-stress reference temperature in all simulations is $\Theta_{\text {ref }}=300 \mathrm{~K}$. Note that the adiabatic staggered scheme is implemented in terms of absolute temperature, so $\Theta$ refers to absolute temperature and $\Delta T=\Theta-\Theta_{\text {ref }}$ refers to the temperature relative to the reference temperature.

Applied load versus deflection curves were extracted for all beams at various temperature variations $\Delta T$. The $R=762 \mathrm{~mm}$ beam (Figure 4 ) shows relatively little variation in load-deflection curve behavior with temperature variation. This plot is representative for the results corresponding to beams with larger curvatures. Figure 5 is representative for beams with smaller curvatures.

The key observation made by examining Figure 5 (and confirmed for the other six beams for which we do not present the results here) is that for each beam there exists a critical temperature below which the beam no longer

experiences snap-through at any load value for a given conservative loading pattern, and exhibits instead a monotonic dependence of the load on deflection. We will simply call this critical value the monotonic temperature. The load-deflection curves for this particular boundary value problem also possess a center point where all the curves obtained for temperatures above the 


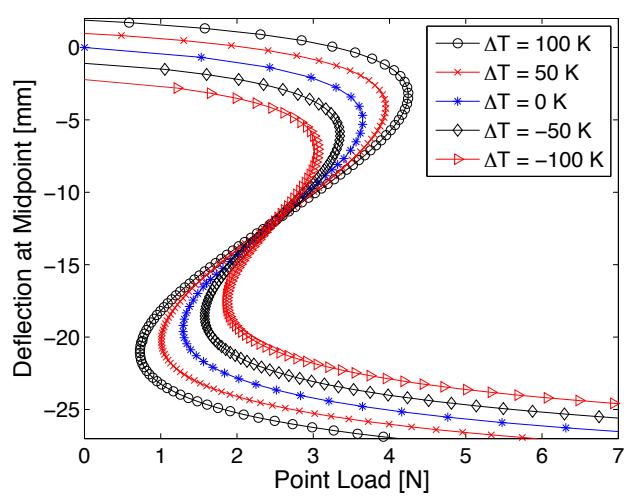

Figure 4: Beam $1(R=762 \mathrm{~mm})$. Pseudo arc-length equilibrium paths for different $\Delta T$.

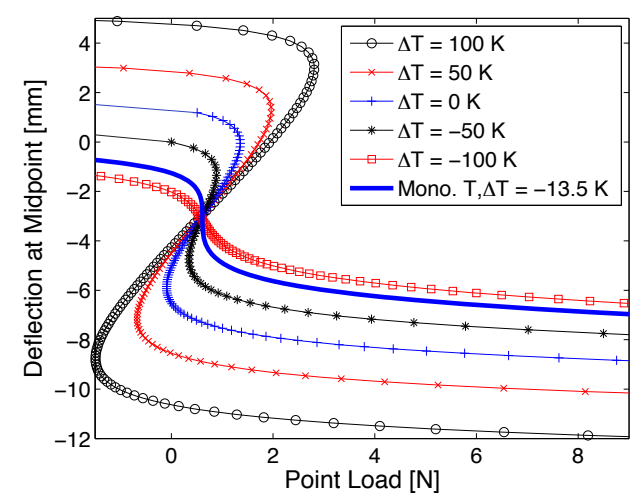

Figure 5: Beam $6(R=3048 \mathrm{~mm})$. Pseudo arc-length equilibrium paths for different $\Delta T$.

monotonic temperature intersect; this center point coincides with the limit point where $\partial P / \partial d=0$ on the monotonic temperature load-deflection curve itself. Results for Beam 1 presented in Figure 4 do not include the monotonic curve since for this particular system the temperature corresponding to it was nonphysical (in a mathematical sense however, it does exist).

The method presented in this paper indirectly estimates the lower bound under the assumption of snap-through to a symmetric solution branch. This method relies on tracing the full equilibrium path and on systematic numerical experimentation to identify the temperature for which the above mentioned path is monotonic. In this approach, we sweep through the range of temperatures of interest and check the monotonicity of the solution.

Although apparently similar results have been known for many years [5], note that the snap-free configuration disclosed in such studies is simply the configuration of an unbuckled, initially flat beam or plate, which obviously will not experience snap-through under any magnitude of lateral loading (if 
no temperature variation is applied). Similarly, the center point in these studies is simply the unloaded initial configuration. Note that if the temperature is raised above a critical buckling temperature, such a member will deform into a curved shape that is itself subject to snap-through [5]. Numerous studies have been devoted to mapping this and the many subsequent post-buckled states $[22,28]$. Our results are distinct from these studies, since the underlying problem is different: here, rather than an initially flat plate or beam which must have some initial thermal deformation to experience snapthrough, we have an initially curved beam with fixed supports that displays snap-through at the zero-stress reference temperature.

Figure 6 indicates that the monotonic temperature approaches $\Theta_{\text {ref }}$ as $\kappa \rightarrow 0$, as expected. For small curvatures the lower bound varies slowly with the curvature. More significant variations are observed for larger curvatures. For very large curvatures the method presented here does not apply since taller arches will buckle asymmetrically. The snap-through loads themselves vary linearly with beam curvature at $\Delta T=0 \mathrm{~K}$, a fact that has been determined analytically at small deformation and which appears to continue to hold at large $\kappa$; however, snap-through loads no longer vary linearly with temperature away from the zero-stress reference temperature (Figure 7). For beams with very low initial curvature, if the temperature is too low, the beam will be straight and in tension and no snap will be experienced.

We can observe several general trends in the results. The relative influence of temperature on the load-deflection behavior of the beam diminishes as the curvature $\kappa=1 / R$ of the beam increases. For the case of $R=254 \mathrm{~mm}$, the effect of even a temperature change of $\Delta T=+100 \mathrm{~K}$ is so small that 


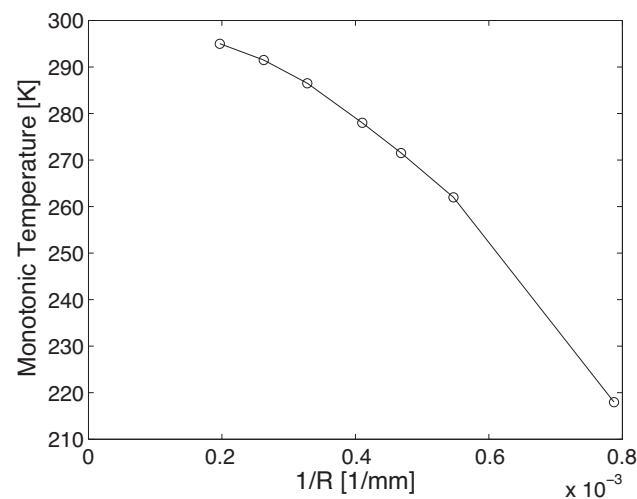

Figure 6: Monotonic temperature varies nonlinearly with curvature.

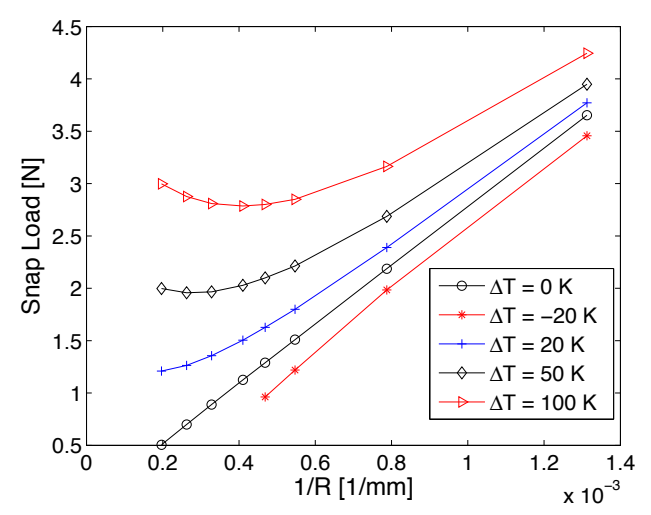

Figure 7: Snap-through load does not vary linearly with curvature away from $\Delta T=0 \mathrm{~K}$.

the load-deflection curves for different temperatures would be difficult to distinguish on a graph; the snap-through load at $\partial P / \partial d=0$ barely changed. By contrast, the influence of temperature on the $R=5080 \mathrm{~mm}$ beam is significant enough that $\Delta T=+100 \mathrm{~K}$ is sufficient to more than triple the snap-through load. This effect can be seen in the decrease in the magnitude of the slope with increasing curvature shown in plots of snap-through load versus temperature in Figure 8. Note that the effect of temperature will be more significant for materials that are more sensitive to temperature, e.g., aluminum.

Figure 9 combines these results and shows the snap-through load as a function of both the geometry (radius) and temperature. The contour line represents the snap-through boundary, i.e., the limit (monotonic) temperature beyond which the beam does not experience snap-through. An alternate method that can be used in obtaining the snap load and the monotonic temperature for shallow arches is presented in [29], which shows a very good 


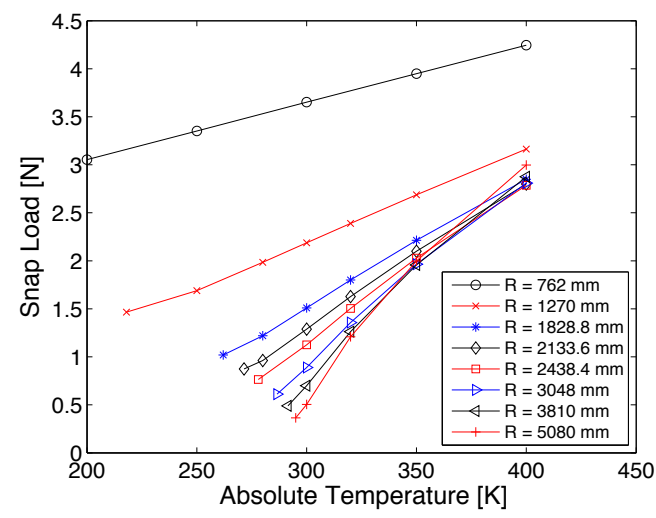

Figure 8: Snap-through loads for all beams at various temperatures.

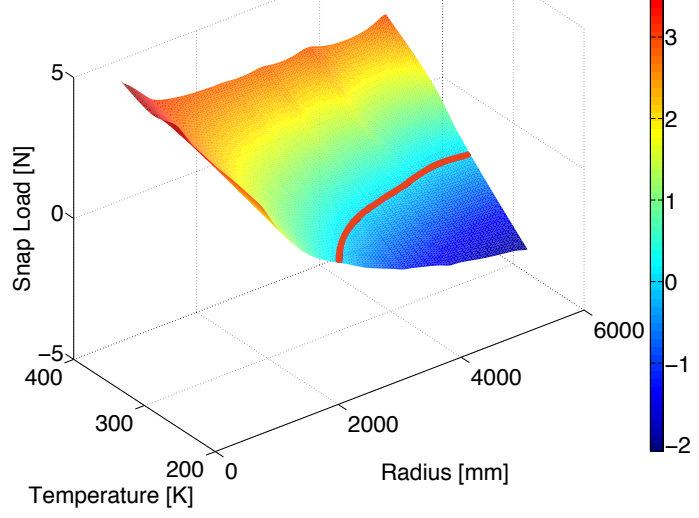

Figure 9: Snap-through loads vs. radius and temperature.

comparison with the results obtained in this paper.

We also perform a study on the effect of beam width and thickness on the snap-through load and the lower bound temperature. The study shows that varying the beam width $(b)$ for the same beam thickness $(h)$ does not influence the lower bound temperature; the snap load, however, increases linearly as the beam width increases (Figure 10). As we vary the beam thickness, the snap load and the lower bound temperature are both higher for thicker beam (Figure 11 and Figure 12).

The pseudo arc-length solution sometimes jumps from the equilibrium path that shows the expected symmetric deformation of the beam (Figure 13) to another path where the beam deforms asymmetrically (Figure 14). This asymmetric buckling mode has been previously discussed [5]. While the second buckling mode is negligible if the initial curvature is small enough [27], this mode clearly cannot be neglected for large initial beam curvatures.

Nevertheless, a unique lower bound on instability for a given beam and 


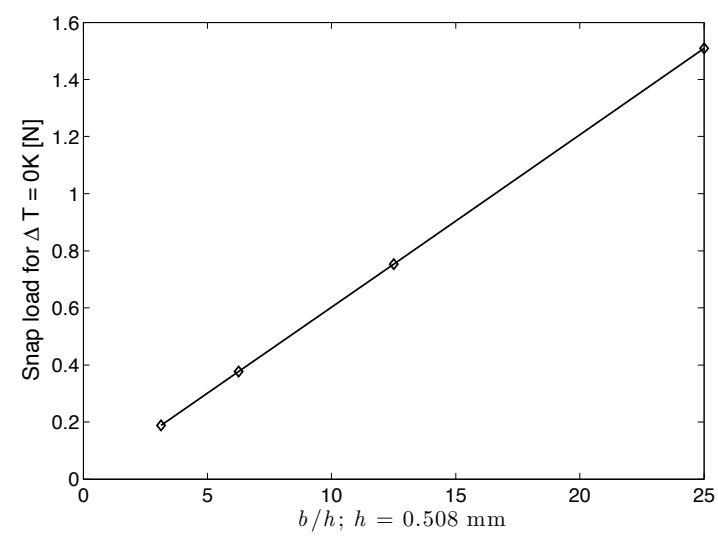

Figure 10: Snap-through loads for varying $\frac{b}{h}$ with $h=0.508 \mathrm{~mm}$ for all beams.

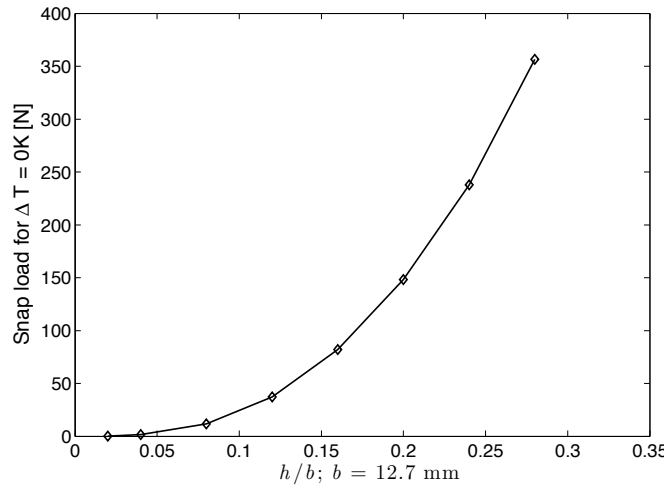

Figure 11: Snap-through loads for varying $\frac{h}{b}$ with $b=12.7 \mathrm{~mm}$ for all beams.

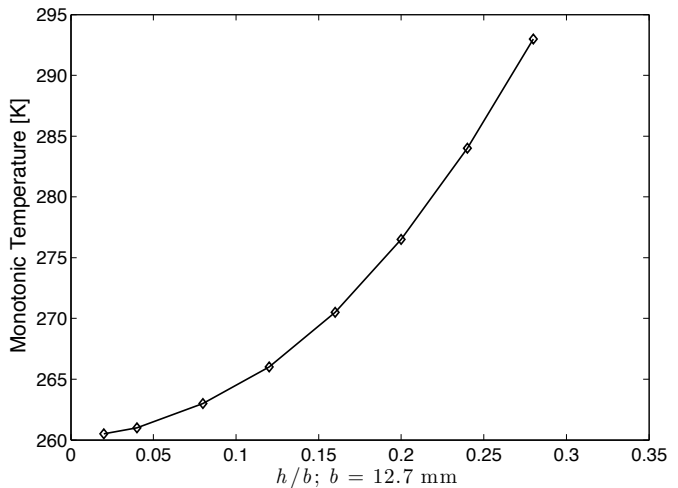

Figure 12: Monotonic temperature for varying $\frac{h}{b}$ with $b=12.7 \mathrm{~mm}$ for all beams.

load pattern can still be established if the second mode only occurs above the monotonic temperature. If the entire unstable region itself is eliminated from the static solution path at the monotonic temperature - including the higher buckling modes - then the lower bound on instability is unique for a given load pattern and initial member shape. No static simulation that we conducted contradicted this conjecture, and dynamic simulation results indicated that higher modes tend to be activated at higher temperatures but 
disappear as temperature is lowered, suggesting that the lower bound is in fact unique.

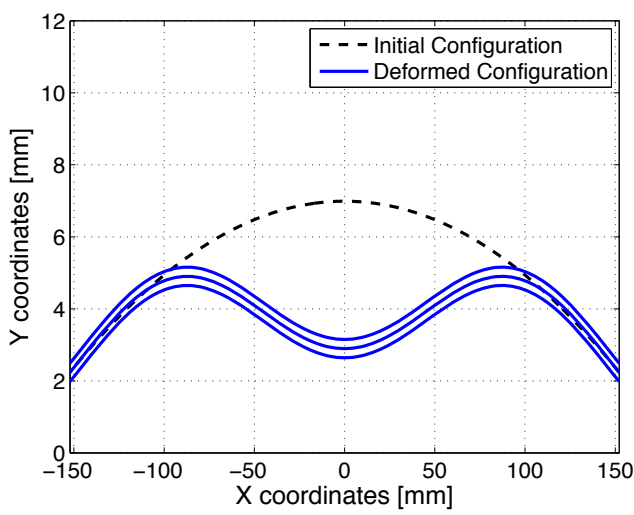

Figure 13: Beam $5(R=2438.4 \mathrm{~mm})$. Symmetric unstable solution; $\Delta T=0 \mathrm{~K}$.

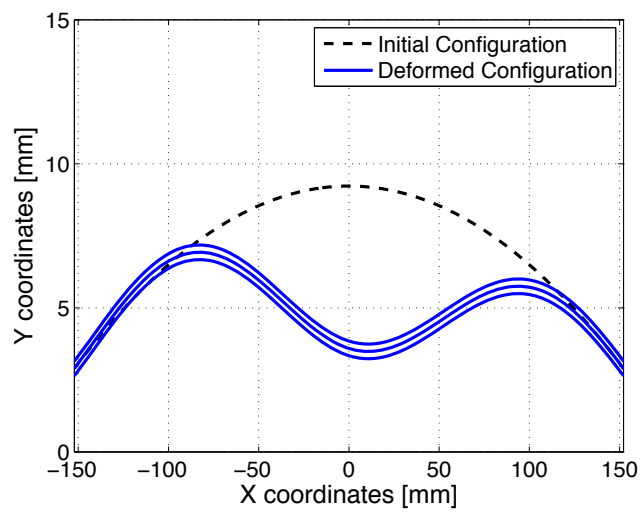

Figure 14: Beam $3(R=1828.8 \mathrm{~mm})$. Unstable asymmetric configuration; $\Delta T=0 \mathrm{~K}$.

\subsection{The Monotonic Temperature and the Energy Derivative Tests}

The coincidence of the limit point of the load-deflection curve at the monotonic temperature with the center point of the graph suggests that there is something special about this point, and inspection of the graphs suggest that this point is likely the inflection point of the curve (Figures 15 and 16.) Numerical evaluation of the derivative $\partial^{2} P / \partial d^{2}$ confirms this. Note that these results are obtained through numerical approximation, therefore the coincidence points are not exact. However, we prove through analytical studies that this hypothesis holds for a simple case [26]. In the case of the symmetric point-loaded beam, we can construct a straightforward physical explanation of the existence of this center point that will help guide our understanding of more general load cases and geometries. 

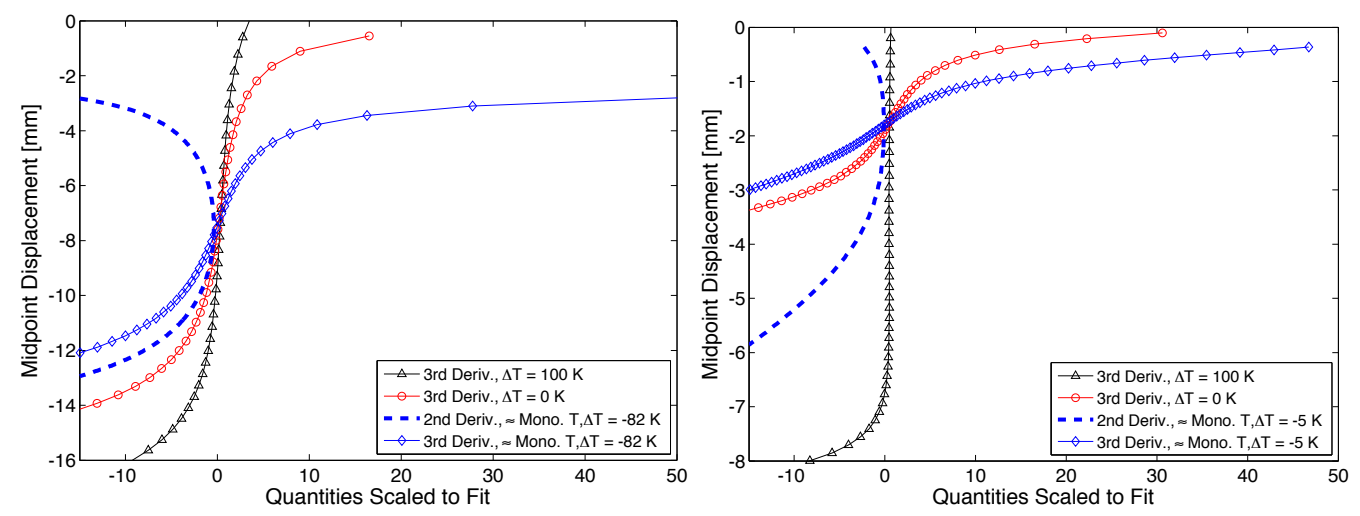

Figure 15: Beam $2(R=1270 \mathrm{~mm})$. Energy derivatives are zero at center point.

Figure 16: Beam $8(R=5080 \mathrm{~mm})$. Observation holds at varied curvatures.

If the beam stays symmetric about the midpoint, the slope of the beam at the midpoint must always be horizontal, and by equilibrium the axial internal force at the midpoint must be equal to the horizontal reaction force at the supports. We intuitively expect that the unstable configuration with the maximum horizontal reaction force is the maximally unstable configuration, that is, the unstable configuration that would move to a stable configuration with the maximum possible kinetic energy relative to all other unstable configurations (if the beam were initially held perfectly fixed at this configuration and then released.)

The maximum horizontal reaction does coincides with the center point of the graphs (Figure 17), indicating that the center point corresponds to the maximally unstable configuration. This does not mean that the horizontal reaction must be less than or equal to zero at the monotonic temperature in order to avoid instability, as one might assume: Figure 17(b) shows that the maximum horizontal reaction can still be compressive at the monotonic temperature. 


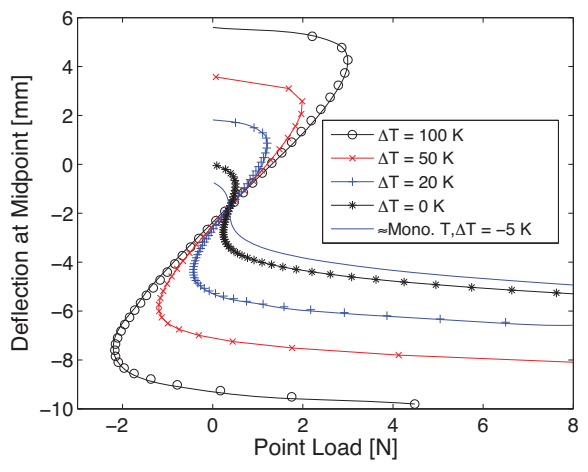

(a) Load-deflection diagram

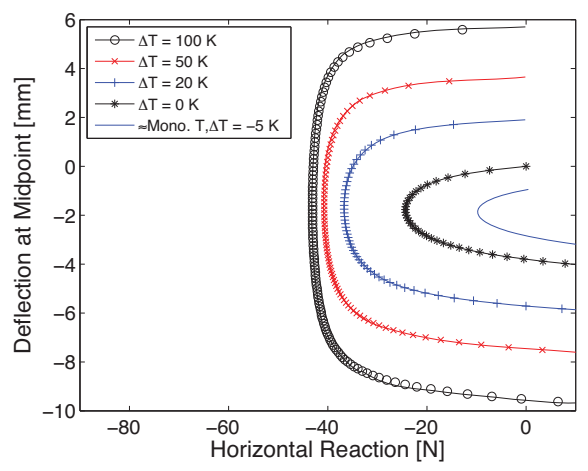

(b) Beam horizonal reaction

Figure 17: Beam $8(R=5080 \mathrm{~mm})$. Maximum horizontal reaction occurs at center point.

The center point is a function of the specific boundary value problem that we chose to explore. We would like to develop a more general test to determine the maximally unstable configuration for problems where the load-deflection curve may not be so well-behaved. The following heuristic argument will point us toward such a test. For this particular problem, the external work can be computed as the integral of the product of the concentrated load $P(d)$ and the deflection at the midpoint $d$.

The second derivative of energy gives the standard static stability test, while the third derivative of energy gives new information that should locate the center point. The third derivative of energy allows us to suggest a mathematical definition of the maximally unstable configuration and the monotonic temperature. Since the value of $\partial^{2} E / \partial d^{2}$ can be considered a measure of the degree of instability present in the system, we would expect that a point where its derivative $\partial^{3} E / \partial d^{3}$ is zero would be an instability extremum that is local to the unstable region. The maximally unstable configuration 
would be the instability extremum in the range where $\partial^{2} E / \partial d^{2}<0$. If we are able to shrink this range by lowering the temperature, then in the limit where the unstable domain shrinks to zero we have both $\partial^{3} E / \partial d^{3}=0$ and $\partial^{2} E / \partial d^{2}=0$ at the same configuration. The temperature that generates this configuration and satisfies these conditions is the monotonic temperature. In other words, the temperature that shrinks the unstable region to a point, for which the maximally unstable $\left(\partial^{3} E / \partial d^{3}=0\right)$ and the minimally stable $\left(\partial^{2} E / \partial d^{2}=0\right)$ configurations are necessarily one and the same, is the monotonic temperature. Note that this is similar to the use of higher derivatives in continuation methods [22], though some simplifications of these more general (and computationally expensive) techniques may be possible for thermomechanical problems, as discussed below.

The energy $E^{\text {int }}$ is obtained from the finite element code, and its numerical derivatives can be approximated by simple difference formulas. We use this data to test the above hypothesis and we do find that the third numerical derivatives of energy are zero at the center point, and the second derivative of energy is zero at the same point for the monotonic temperature (Figure 15). This observation holds for beams of significantly different curvatures (Figure 16).

For the case of the distributed load (Figure 2b), a global center point no longer exists (Figure 18). The curves for lower temperatures possess a center point but the curves at elevated temperatures do not pass through it. Similar to the case of the concentrated load, the curved beam acts like an arch under distributed load, resisting primarily through axial stiffness rather than through weaker flexural stiffness. However, this "arch" is not 


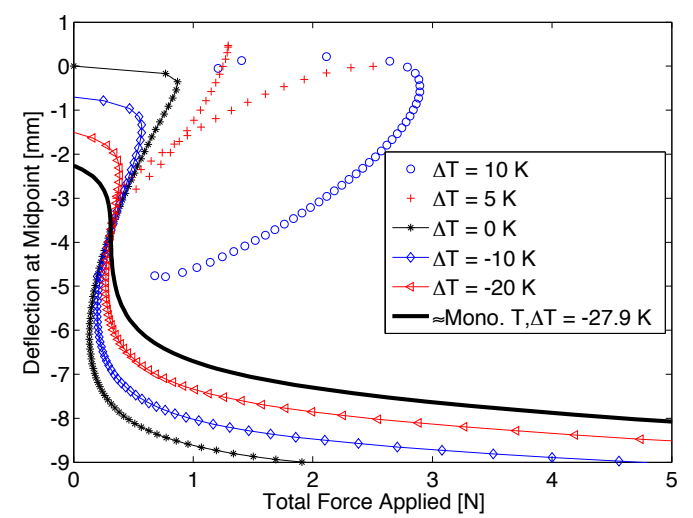

Figure 18: Beam $5(R=2438.4 \mathrm{~mm})$. Load-deflection curves for distributed loads: no center point at elevated temperatures.

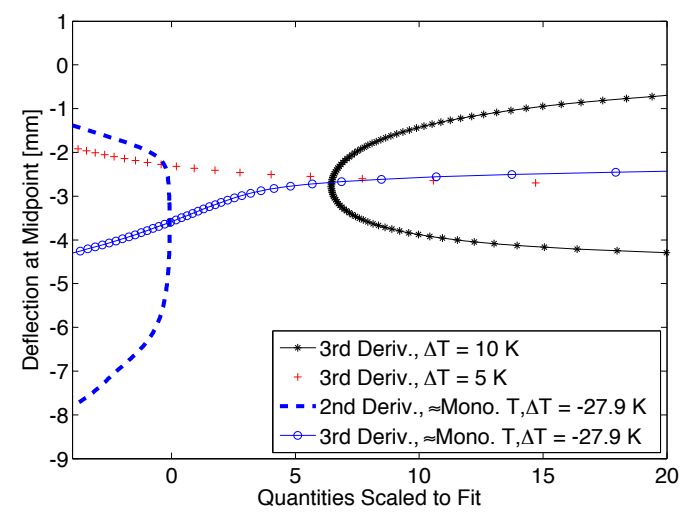

Figure 19: Beam $5(R=2438.4 \mathrm{~mm})$. Energy derivatives are still zero at monotonic temperature despite absence of a global center point.

geometrically perfect and suffers from local flexural buckling (Figures 20(a) to 20(c)) at load values below those achieved in the case of the concentrated load.

These higher buckling modes may deflect upward at the midpoint even as global stability is lost, so we can no longer assume that $\partial P / \partial d=0$ indicates a snap-through point, at least at elevated temperatures. Similarly, $\partial^{3} E / \partial d^{3}=$ 0 does not occur in the distributed load case at high temperatures and can therefore no longer be related to the maximally unstable configuration as it can in the concentrated load case. Nonetheless, the energy derivatives are still zero at the monotonic temperature (Figure 19).

The distributed load case reinforces the earlier observation that the value of the monotonic temperature depends on the pattern of applied load: for the $R=2438.4 \mathrm{~mm}$ beam, the symmetric point load leads to a monotonic temperature of $\Delta T=-22 \mathrm{~K}$, while for the distributed load case the monotonic temperature is approximately $\Delta T=-28 \mathrm{~K}$. The decreased monotonic 


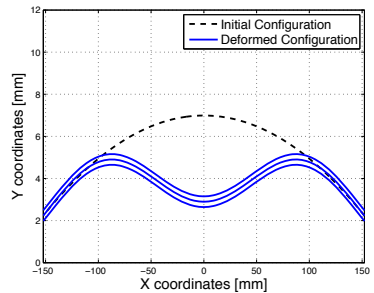

(a) $\Delta T=0 \mathrm{~K}$

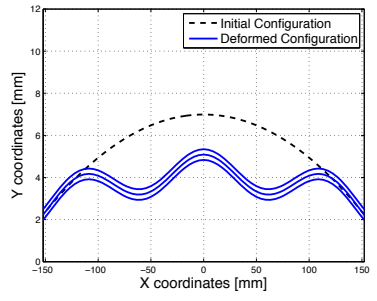

(b) $\Delta T=5 \mathrm{~K}$

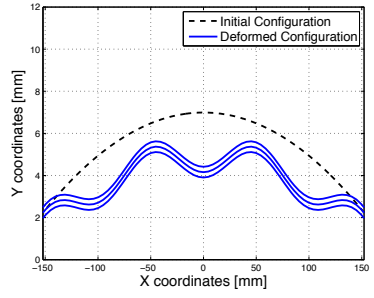

(c) $\Delta T=10 \mathrm{~K}$

Figure 20: Beam $5(R=2438.4 \mathrm{~mm})$ with distributed load.

temperature for the distributed load case indicates that it is possible to find a loading such that a "worst-case" monotonic temperature is obtained and a lower bound on the thermoelastic instability for all possible loadings of a system is established. This requires the solution of an inverse problem to determine the worst-case loading, which entails more complexity than is typically worth the effort, especially if the actual load patterns a structure is subject to can be known in advance with reasonable certainty. This concept is not developed further in this paper, and all monotonic temperatures reported are limited to the specific load cases considered.

In all these examples, due to symmetry, the derivatives with respect to $d$ (the midpoint deflection) provide clear information about the stability of the system. In general, there will not be a simple line of symmetry that will allow us to obtain meaningful information, as is apparent from the higher buckling mode load-deflection curves in Figure 20. It would be preferable to develop an analytical test for the third derivative of energy that will let us circumvent the process of taking numerical derivatives entirely and allow us to determine the monotonic temperature for any given system.

Before assuming that the monotonic temperature obtained from static 
simulations is truly effective in eliminating snap-induced vibrations, we must first demonstrate that it eliminates snap-through in dynamic systems. The next section is devoted to establishing the validity of generalizing from the static, mechanical-only solution to the dynamic, thermomechanically coupled case. Then, we outline a test for establishing the monotonic temperature for arbitrary loading and member geometries in the final section.

\section{Dynamic Simulation of Snap-Through}

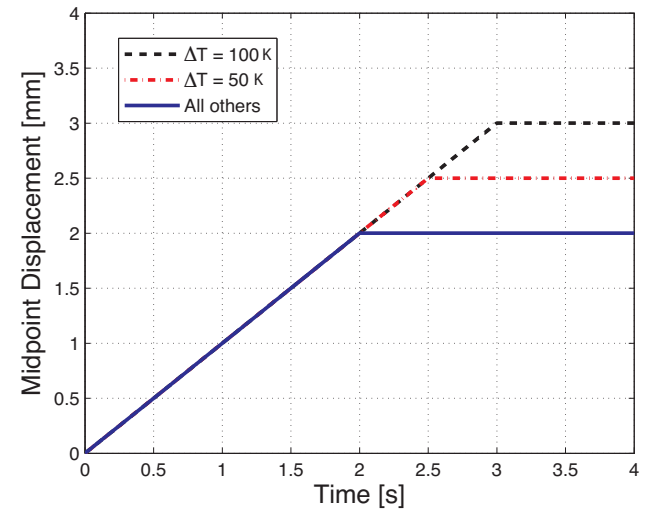

Figure 21: Ramp load target values.

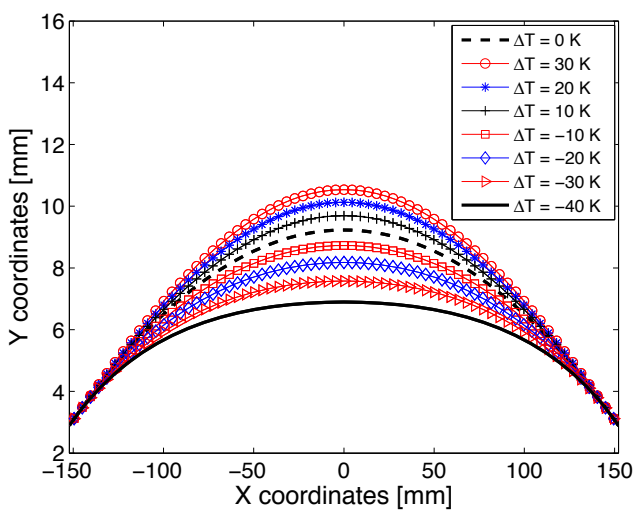

Figure 22: Beam shapes at varied initial temperatures.

In order to determine if the monotonic temperature estimated via static simulations successfully eliminates snap-through, dynamic simulations are conducted. We present here the results obtained for one beam only $(R=$ $1828.8 \mathrm{~mm}$ ), with the same reference temperature $\Theta_{\text {ref }}=300 \mathrm{~K}$ used in the static simulations. Consequently, the beam number is no longer mentioned in the figure captions. This particular beam has a large initial curvature but still possesses an estimated monotonic temperature that could be easily obtained 
in the laboratory $\left(\Delta T=-38 \mathrm{~K}\right.$ relative or $\Theta=-11^{\circ} \mathrm{C} / 12^{\circ} \mathrm{F}$ absolute). A concentrated force is applied at an effectively quasi-static rate at midspan and then held constant at a fixed target value greater than the static snapthrough load $P_{s}$. The target load is $P=2.0 \mathrm{~N}$ for temperatures below $T=30 \mathrm{~K}$, but the increase in snap-through load at increased temperature necessitated higher target loads of $P=2.5$ and $3.0 \mathrm{~N}$ for $T=50$ and $100 \mathrm{~K}$ respectively (Figure 21).

Initial static simulations were conducted to obtain the thermally-deformed initial configuration of the beam at each target temperature prior to dynamic simulation (Figure 22). The initial temperature was applied as a perfectly uniform nodal initial condition and the surfaces of the beam were treated as perfectly insulated. Though this zero-heat-flux boundary condition is not feasible in an experimental setting, it allows us to isolate the temperature change due to thermomechanical coupling in our simulations. Purely mechanical dynamic simulations [20] had earlier indicated that a problemspecific critical time step of $\Delta t=10^{-4} \mathrm{~s}$ or smaller must be used for accurate modeling of snap-through. Values of $\Delta t$ above this critical value could lead to spurious solutions that are clearly nonphysical but nonetheless numerically converged.

The monotonic temperature estimated from static simulations successfully eliminated snap-through from dynamic simulations at quasi-static load rates. The maximum amplitude of displacement does clearly approach zero as the temperature approaches the monotonic temperature, and effectively reaches zero for $\Delta T=-50 \mathrm{~K}$ (Figure 23). The maximum displacement approaches a limit as the temperature increased; however, the maximum kinetic 
energy over all time values continues to increase linearly as the temperature increases (Figure 24). This "excess" kinetic energy is present in asymmetric deformation modes not apparent from examination of the midpoint displacement alone.

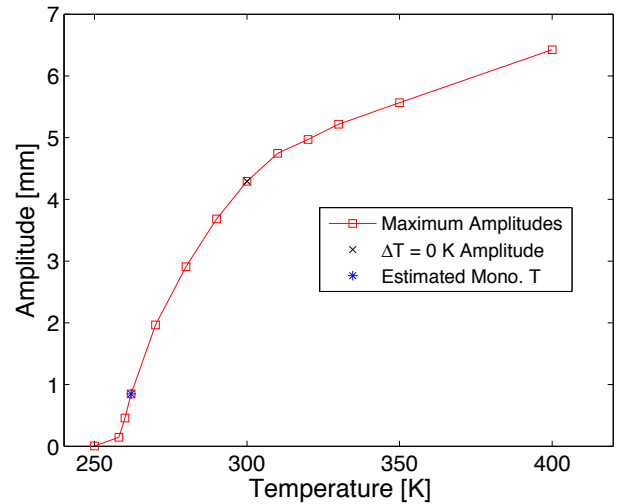

Figure 23: Maximum ampltiude of vibrations at various temperatures. Vibrations disappear near monotonic temperature and level off at elevated temperature.

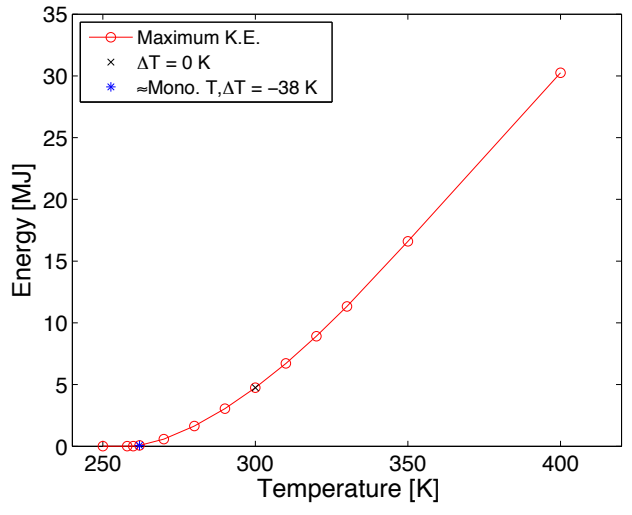

Figure 24: Maximum kinetic energy at various temperatures. Kinetic energy disappears near monotonic temperature and increases linearly at elevated temperatures.

Note that the ramp load continues to increase prior to reaching a constant value after snap-through, hence, the centerline of the vibrations moves downward on the plot prior to $t=2.0 \mathrm{~s}$ (Figure 26). This component of the vibration must be filtered out in order to determine the amplitudes of vibration correctly. This was done by fitting a sixth-order polynomial to the post-snap midpoint displacement time history data and finding the value of the maximum difference between the smooth curve and the displacement.

The y-direction displacement at the midpoint is represented in Figures 25 to 27 as a function of time for various initial temperatures and the static simulation results are superposed at the same scale. Thermoelastic damping is 
clearly apparent in these plots; by comparison, purely mechanical simulations of snap-through using the trapezoidal Newmark method showed no damping of the post-snap vibrations, as is expected for this energy-conserving time integration algorithm. Visual inspection of the graphs also indicates that the large-amplitude vibrations characteristic of snap-through are strongly attenuated below the monotonic temperature of $\Delta T=-38 \mathrm{~K}$ (Figure 26), dropping to nearly zero at $\Delta T=-50 \mathrm{~K}$ (Figure 27).

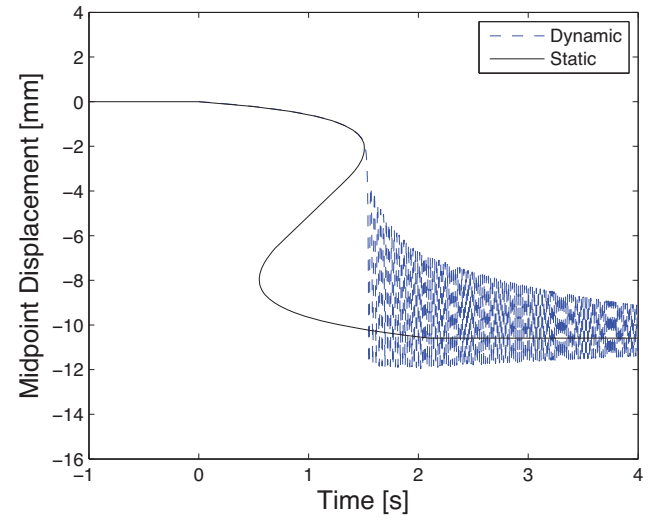

Figure 25: Displacement at $\Delta T=0 \mathrm{~K}$.

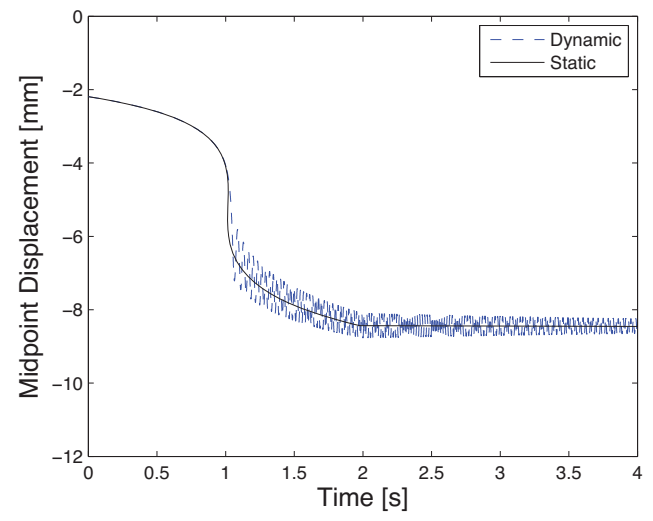

Figure 26: Displacement at $\Delta T=-38 \mathrm{~K}$.

A measure of the asymmetry of vibration can be constructed by plotting the difference between the $\mathrm{y}$-direction displacement $w(x)$ at the points $x=L / 4$ and $x=3 L / 4$. This difference will be zero if the vibration is symmetric, or otherwise can give an estimate of the frequency and magnitude of the asymmetric vibration. For $\Delta T=0 \mathrm{~K}$ and below, the post-snap vibration remains perfectly symmetric for the beam considered. For $\Delta T=30 \mathrm{~K}$, the post-snap vibration is initially symmetric, but after the load reaches a constant value the response develops an antisymmetric vibration (Figure 28). This vibration damps out relatively quickly at $\Delta T=30 \mathrm{~K}$, but at $\Delta T=50$ 
$\mathrm{K}$ a similar mode of vibration is present at a higher amplitude and for a longer duration (Figure 29). For $\Delta T=100 \mathrm{~K}$, the beam buckles asymmetrically immediately prior to the static snap-through load and remains in a fully asymmetric mode after snap-through (Figure 30).

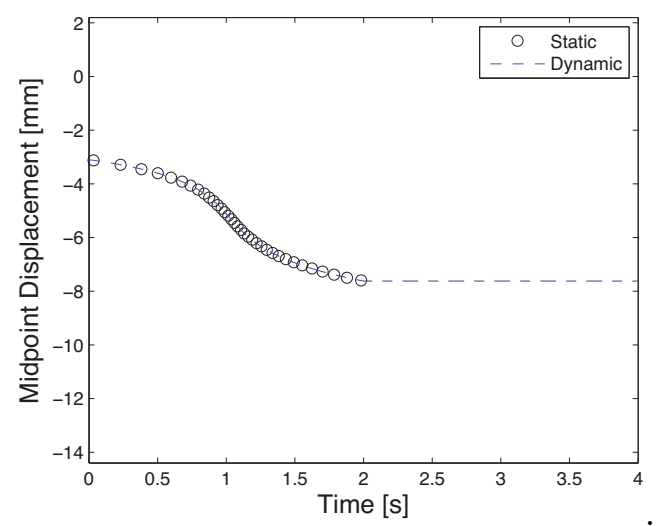

Figure 27: Displacement at $\Delta T=-50 \mathrm{~K}$.

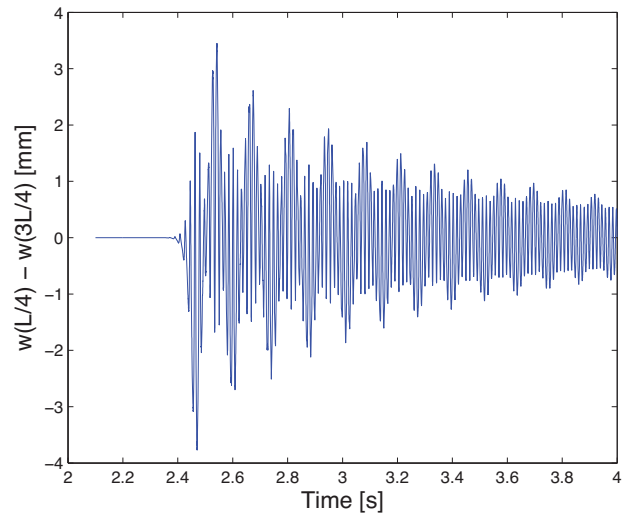

Figure 29: Low-frequency asymmetric vibration sustains longer for $\Delta T=50 \mathrm{~K}$.

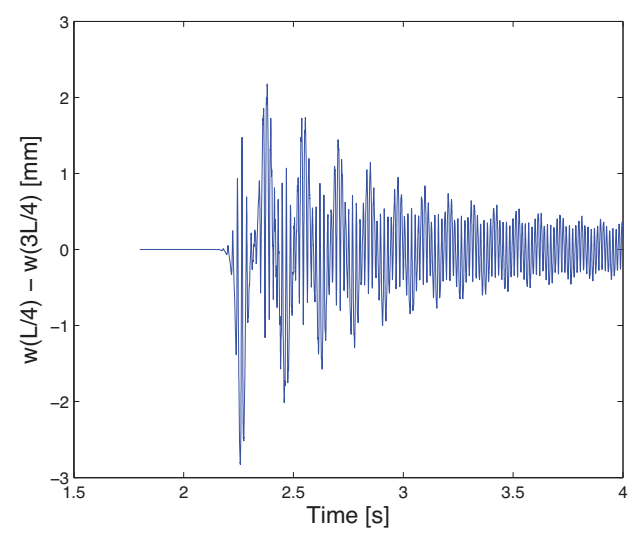

Figure 28: Low-frequency asymmetric vibration damps out for $\Delta T=30 \mathrm{~K}$

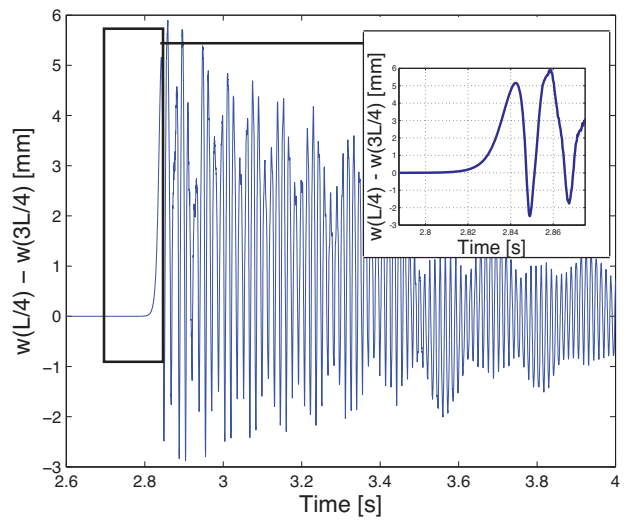

Figure 30: Beam buckles asymmetrically prior to snap-through at $\Delta T=100 \mathrm{~K}$.

Qualitatively, the $\Delta T=30 \mathrm{~K}$ and $\Delta T=50 \mathrm{~K}$ cases combine a sym- 
metric mode (Figure 31a) and an extremely low frequency asymmetric mode (Figure $31 \mathrm{~b}$ ). The $\Delta T=100 \mathrm{~K}$ case buckles asymmetrically prior to snapthrough (Figure 32a) and remains in a high-frequency asymmetric mode that oscillates at an angle to the vertical following buckling (Figure 32b). As this asymmetric mode is damped, a new mode emerges (following $t=3.4 \mathrm{~s}$ ) similar to the low-frequency asymmetric mode encountered at $\Delta T=30 \mathrm{~K}$ and $\Delta T=50 \mathrm{~K}$.

Unloading the beam from an initially stationary deformed configuration reveals that the vibration about the initial undeformed configuration is of significantly lower amplitude than the vibration about the deformed configuration. Although the initial post-snap unloading response begins as a symmetric vibration, it develops a small asymmetry that quickly grows in magnitude. While the initial transient mode shape is essentially similar to the low-order symmetric mode of the loading response (Figure 33a), inspection of the deformed configurations reveals that the asymmetric mode behaves like a low-frequency "wave" reflecting back and forth between the supports (Figure 33b). Figure 34 shows the loading and unloading responses.

These various modes interact nonlinearly in the finite-deformation model, and the resulting transient behavior can become difficult to quantify, especially as higher modes are activated and the post-snap motion increases in complexity. However, the higher modes diminish with decreasing temperature and eventually disappear at the monotonic temperature, making the monotonic temperature a solid point of reference in an otherwise convoluted post-buckling regime.

The monotonic temperature also holds for the distributed load case. The 
a.)

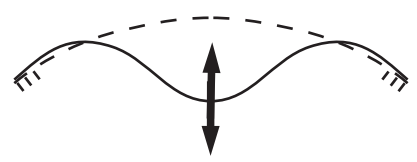

b.)

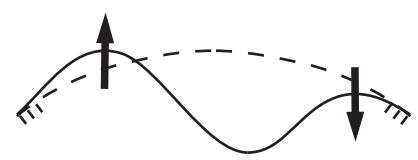

Figure 31: Mode shapes for $\Delta T=30$ and $50 \mathrm{~K}$ (a) Symmetric and (b) Asymmetric.

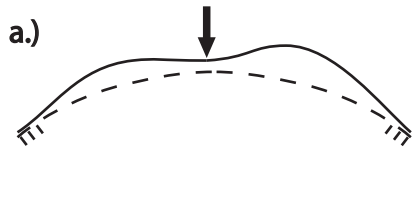

b.)

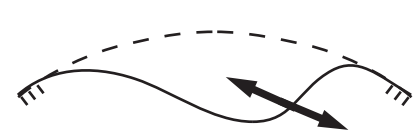

Figure 32: $\Delta T=100 \mathrm{~K}(\mathrm{a})$ Initial asymmetric buckling and (b) Asymmetric "sideways" postbuckling mode.

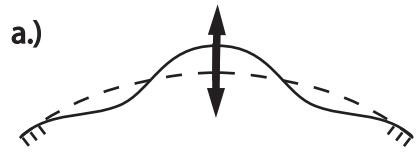

b.)

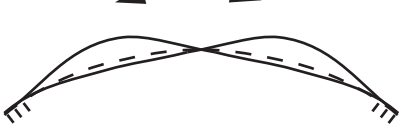

$R=2438.4 \mathrm{~mm}$ beam displays the same sort of asymmetric buckling prior to the static snap-through load at $\Delta T=0 \mathrm{~K}$ under distributed loading that is seen at $\Delta T=100 \mathrm{~K}$ for the $R=1828.8 \mathrm{~mm}$ beam under concentrated loading. At the estimated monotonic temperature nonetheless, post-snap vibration are attenuated as effectively in the distributed load case as they are in the concentrated load case (Figure 35).

There are some caveats on the generality of the monotonic temperature. The softening response seen in the static solution paths even for temperatures below the monotonic temperature can still lead to snap-like behavior if the loading rate is high enough to force the system to dynamically jump off the static solution curve as the system softens with increasing load. More generally, the system must be dissipative for the temperature to remain below the monotonic temperature. This is true in our case, but it is no longer valid if body heat sources or applied surface heat fluxes are present. We are therefore limited to saying that the monotonic temperature provides a lower 


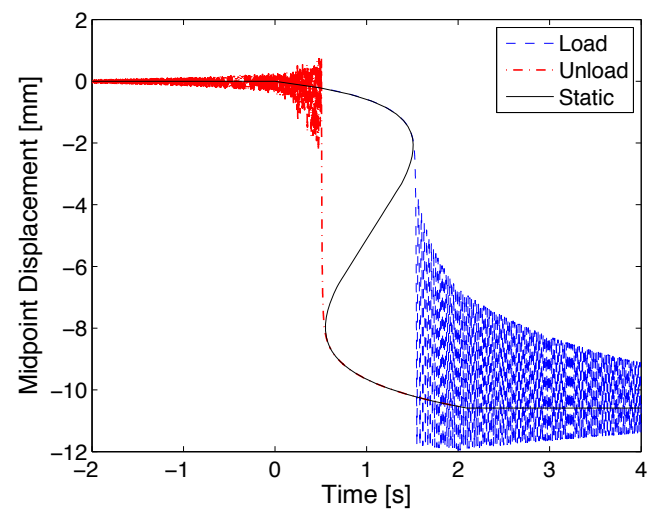

Figure 34: Beam 3 $(R=1828.8 \mathrm{~mm})$. Asymmetry of loading and unloading response at $\Delta T=0 \mathrm{~K}$.

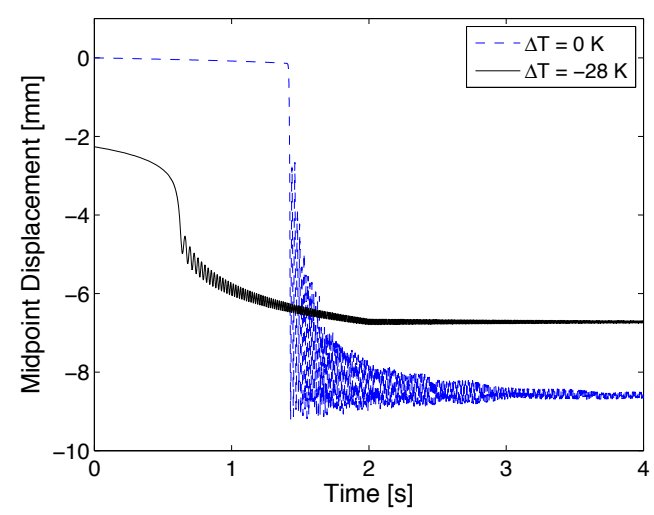

Figure 35: Beam $5(R=2438.4 \mathrm{~mm})$. Static monotonic temperature estimate holds for distributed load case.

bound below which snap-through will never occur at any applied load level if the loading rate is effectively quasi-static and the system is dissipative.

The monotonic temperature estimated from static simulations is not perfectly precise; it does not completely eliminate the oscillations even for dynamic systems with effectively quasi-static loading. Some of this error is due to the simple approach that was undertaken to find this temperature, where the monotonicity of the curve was determined only approximately. Another source for the discrepancy is due to the physical fact that the ramp loading can never be truly quasi-static in a dynamic simulation. The amplitude of vibration will go to zero nearer the monotonic temperature (refer to Figure 23) if the loading rate is decreased, but there will always exist some small dynamic jump as the system approaches the limit point on the static load-deflection curves.

One additional practical observation can be made. We have modeled the curved beam with an edge that is free to move in the z-direction (out-of-plane) 
between the fixed supports. In many practical engineering applications, such as stressed-skin monocoque aircraft construction, the z-direction will be restrained as well, and we expect this additional boundary condition to have a stabilizing effect. These additional boundary conditions increase the snapthrough load (Figure 36) as well as the monotonic temperature itself (Figure 37). The monotonic temperatures obtained from the static simulations without these boundary conditions are therefore conservative values relative to systems that include such constraints.

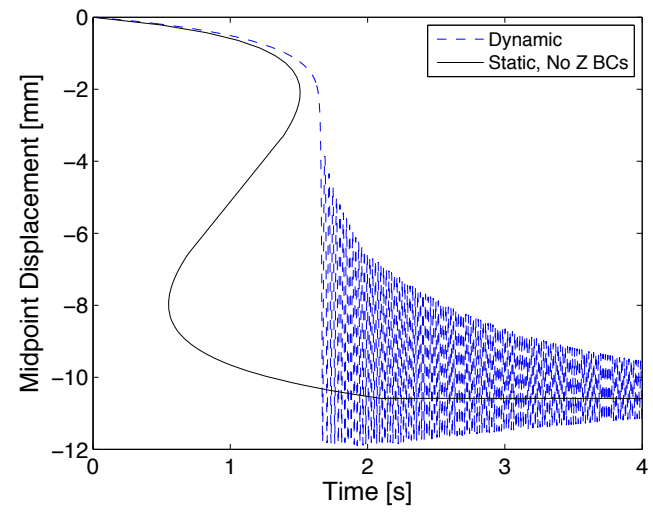

Figure 36: Beam 3 $(R=1828.8 \mathrm{~mm})$. Full z-direction edge boundary condition increases snap-through load.

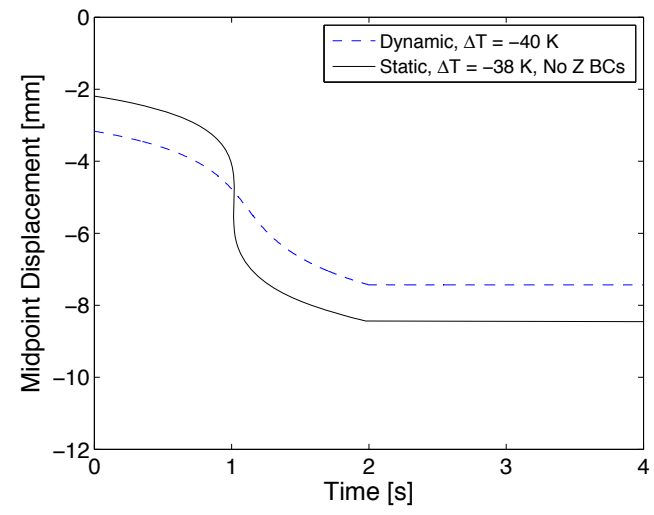

Figure 37: Beam $3(R=1828.8 \mathrm{~mm})$. Full z-direction edge boundary condition increases monotonic temperature.

Note that, in the static simulations, we have omitted heat conduction and treated temperature as a purely mechanical expansion or contraction of the material, which provided a valid estimate of the snap-free monotonic temperature for the dynamic thermomechanically coupled case. This analogy between mechanical strain and thermal strain indicates that an applied mechanical prestress will have a mechanical effect similar to lowering the temperature. For example, for the unloaded $R=1270 \mathrm{~mm}$ beam, the reaction 
at the supports has no vertical component and has a horizontal component of $P_{x}=9.42 \mathrm{~N}$ at the monotonic temperature $\Delta T=-82 \mathrm{~K}$; the horizontal reaction is $P_{x}=15.4 \mathrm{~N}$ for $\Delta T=-100 \mathrm{~K}$. Given the out-of plane depth of $d=28.7 \mathrm{~mm}$ and beam thickness $t=1.27 \mathrm{~mm}$, and assuming a uniform stress over the cross-section, the axial stress in the beam is therefore only $\sigma / d t=0.4225 \mathrm{MPa}$ for $\Delta T=-100 \mathrm{~K}$, which is only a minute fraction of the yield stress of $\sigma_{y}=250 \mathrm{MPa}$ for A36 structural steel. Although $\Delta T=-100 \mathrm{~K}$ is clearly an unrealistic temperature, $\sigma=0.4225 \mathrm{MPa}$ is a perfectly realistic prestress. Consequently, an applied mechanical prestress will have a mechanical effect similar to lowering the temperature, and therefore we could eliminate quasi-static snap-through by applying a purely mechanical pre-tension sufficient to achieve a tensile strain identical to or greater than that produced by the monotonic temperature. Moreover, it is also possible to apply additional initial strain sufficient to cancel the effects of thermal expansion, so that a heated beam can be "snap-proofed" for temperatures below a given elevated target temperature.

In practical aerospace applications, significant prestress is introduced to thin structural members to provide increased structural stiffness, independent of any thermal considerations. This prestress is sufficient to introduce tensile strains equivalent or greater than those introduced by the monotonic temperature, as is apparent from the above example. It is therefore unlikely that typical aerospace structures will experience snap-through under standard operating conditions. However, as the operating conditions of the structure become more severe, as in hypersonic flight, much larger thermal loads can be expected. Additional mechanical pre-tension could therefore prove 
useful in providing additional protection against snap-through in aerospace structures in extreme operating environments, although more research would be required to determine the effectiveness, applicability, and limitations of this approach.

\section{Maximum Instability and Bounds on Snap-Through}

In the previous sections, we have established that (1) the monotonic temperature represents a lower bound on static instability in curved beams subject to snap-through, and (2) for the simple symmetric beam with a concentrated load, the second and third derivatives of energy with respect to the midpoint displacement are both zero at the snap-through point at this temperature. We surmised that the unstable region shrinks as the temperature is lowered until it becomes a point at the monotonic temperature, and that at this point the minimally stable configuration $\left(\partial^{2} E / \partial d^{2}=0\right)$ and maximally unstable configuration $\left(\partial^{3} E / \partial d^{3}=0\right)$ are identical. This shrinkage of the unstable region to a point as temperature is lowered is illustrated conceptually in Figure 38. We also observed that, although multiple modes may exist corresponding in some cases to multiple paths within the unstable region (Figure 39), in static simulations these modes tend to disappear as the monotonic temperature is approached.

These observations suggest a mathematical definition of the monotonic temperature. We do not attempt to prove the following conjectures, but the simulation results support them. If we are able to shrink the unstable region along the equilibrium path of our system by altering some parameters (such as temperature), then the configuration where the critical parameters 


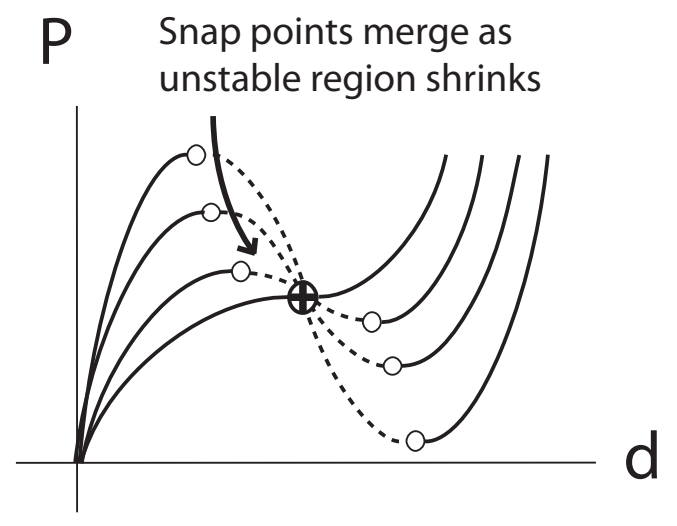

Figure 38: Conceptual illustration of lower bound.

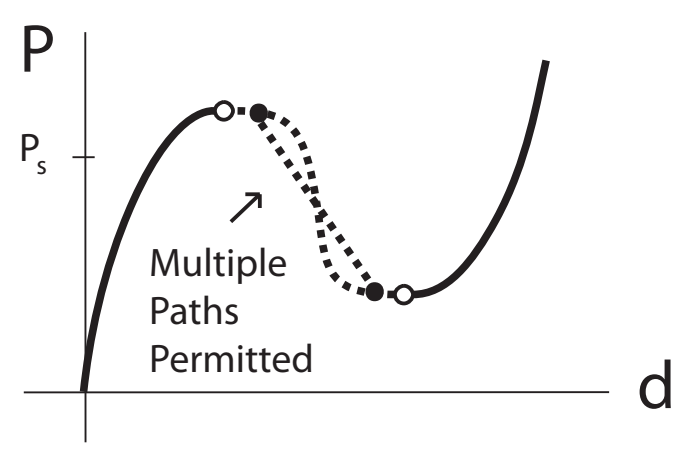

Figure 39: Multiple paths within the unstable region.

are such that snap-through no longer occurs is exactly the point where the minimally stable configurations at the boundaries of the unstable region $\mathrm{co}$ incide with the maximally unstable configuration. Such conditions should therefore give us a set of equations that provide a bound of the domain of unstable behavior. Without attempting to prove them, we state here these conditions.

Conjecture : Let $d$ be the displacement field in a continuum, and let $\mathbf{p}$ be a vector of bifurcation parameters, which control the size of the unstable region. Assume that the system under consideration has only one unstable region. Let $G, A, B$ be the first, second and third derivatives of the energy. The unstable region ceases to exist for the particular configuration $\left(d^{*}, \mathbf{p}^{*}\right)$ at which, for all virtual displacement fields $\delta d$, the functionals $G, A$ and $B$ are null: $G\left(d^{*}, \mathbf{p}^{*}, \delta d\right)=0, A\left(d^{*}, \mathbf{p}^{*}, \delta d\right)=0$, and $B\left(d^{*}, \mathbf{p}^{*}, \delta d\right)=0$.

If we use a finite element approximation of the functionals from a continuum problem, we obtain the version of the above conditions in matrix form: $\mathbf{G}\left(\mathbf{d}^{*}, \mathbf{p}^{*}\right)=0, \operatorname{det} \mathbf{A}\left(\mathbf{d}^{*}, \mathbf{p}^{*}\right)=0$, and $\operatorname{det} \mathbf{B}\left(\mathbf{d}^{*}, \mathbf{p}^{*}\right)=0$. In this discretized 
form, $\mathbf{G}$ is a vector (the residual vector in finite element methods), $\mathbf{A}$ is a second-order tensor (the stiffness matrix), $\mathbf{B}$, is third-order tensor, and $\mathbf{d}^{*}$ is a vector (of nodal displacements).

A simple nonlinear Timoshenko beam model demonstrates the utility of these conjectures and also demonstrates why limited-deflection models, such as the von Karmann equations, are a priori incapable of determining the monotonic temperature. The interested reader is referred to [26] for the detailed derivation corresponding to the beam model. Unlike the fully nonlinear equilibrium equations, the third variational derivative of the linearized beam equations is identically zero. In taking the Taylor series approximation of the fully nonlinear Timoshenko beam equilibrium equations we have lost information, and, as discussed in [26], the information we have lost from the third derivative of energy is exactly the information we require to be able to pinpoint the nontrivial snap-free configuration that exists at the monotonic temperature. The von Karmann equations therefore do not disclose the existence of the nontrivial solution precisely because the information that is needed to determine it has been omitted a priori.

\section{Conclusions}

We demonstrated the existence of a non-trivial curved beam configuration and a corresponding critical temperature, lowered with respect to the refer-

ence temperature, that eliminates the occurrence of dynamic snap-through under quasi-static loading rates. This monotonic temperature can be estimated from static solution paths with reasonable accuracy, even for beams of large initial curvature. An analytical test based on the third variational 
derivative of the energy that could be useful in determining the monotonic temperature for members of general geometry and load patterns is also suggested. The failure of limited-deformation thermal beam and plate models such as the von Karmann equations to disclose the existence of a nontrivial snap-free solution is shown to be a consequence of the omission of information about the third variational derivative of energy from these lower-order theories.

The analogy between purely mechanical contraction and thermal strain that holds for these numerical experiments suggests that an applied mechanical prestress that introduces an initial strain equivalent to the strain induced by the monotonic temperature eliminates the possibility of quasistatic snap-through in beams of large initial curvature. Increasing the initial prestress in thin-walled structures using values determined from this procedure could therefore provide a simple method of protecting against large amplitude snap-through vibrations encountered in by aircraft and spacecraft in extreme operating environments. Although this method would introduce additional stresses into the supporting members and therefore has limitations depending on the requirements of the structure, it is simpler than other methods that have been proposed in the literature [30]. Further research would be needed to determine the potential effectiveness and practical limitations of this proposed method of alleviating dynamic snap-through.

\section{Acknowledgements}

The authors would like to thank Professor Lawrence Virgin (Duke University) for his valuable comments and suggestions. 
The work has been funded in part by AFOSR under the grant no. FA955009-1-0201. This support is greatly appreciated.

\section{References}

[1] K. Murphy, L. Virgin, S. Rizzi, Characterizing the dynamic response of a thermally loaded, acoustically excited plate, Journal of Sound and Vibration 196 (1996) 635-658.

[2] W. Y. Tseng, J. Dugundji, Nonlinear vibrations of a beam under harmonic excitation, Journal of Applied Mechanics, Transactions ASME 37 Ser E (2) (1970) $292-297$.

[3] W. Kreider, A. Nayfeh, Experimental investigation of single-mode responses in a fixed-fixed buckled beam, Nonlinear Dynamics 15 (2) (1998) $155-177$.

[4] K. Murphy, L. Virgin, S. Rizzi, Experimental snap-through boundaries for acoustically excited, thermally buckled plates, Experimental Mechanics 36 (4) (1996) $312-317$.

[5] H. Irschik, Large thermoelastic deflections and stability of simply supported polygonal panels, Acta Mechanica 59 (1) (1986) 31 - 46.

[6] P. Ribeiro, E. Manoach, The effect of temperature on the large amplitude vibrations of curved beams, Journal of Sound and Vibration 285 (4-5) (2005) $1093-1107$. 
[7] Y.-L. Yeh, Chaotic and bifurcation dynamic behavior of a simply supported rectangular orthotropic plate with thermo-mechanical coupling, Chaos, Solitons and Fractals 24 (5) (2005) 1243 - 1255.

[8] D. Liaw, Nonlinear supersonic flutter of laminated composite plates under thermal loads, Computers and Structures 65 (5) (1997) 733 - 740.

[9] M. Ganapathi, T. Prakash, Supersonic flutter characteristics of functionally graded flat panels including thermal effects, Composite Structures 72 (1) (2006) 10 - 18, URL http://dx.doi.org/10.1016/j. compstruct.2004.10.007.

[10] J.-M. Dhainaut, X. Guo, C. Mei, S. M. Spottswood, H. F. Wolfe, Nonlinear random response of panels in an elevated thermal-acoustic environment, Journal of Aircraft 40 (4) (2003) 683 - 691.

[11] Y.-L. Yeh, C.-K. Chen, H.-Y. Lai, Chaotic and bifurcation dynamics for a simply supported rectangular plate of thermo-mechanical coupling in large deflection, Chaos, Solitons and Fractals 13 (7) (2002) 1493 - 1506.

[12] L. Librescu, W. Lin, M. Nemeth, J. Starnes Jr., Vibration of geometrically imperfect panels subjected to thermal and mechanical loads, Journal of Spacecraft and Rockets 33 (2) (1996) 285 - 291.

[13] J.-S. Park, J.-H. Kim, S.-H. Moon, Thermal post-buckling and flutter characteristics of composite plates embedded with shape memory alloy fibers, Composites Part B: Engineering 36 (8) (2005) 627 - 636. 
[14] I. Lee, D.-M. Lee, I.-K. Oh, Supersonic flutter analysis of stiffened laminated plates subject to thermal load, Journal of Sound and Vibration 224 (1) (1999) $49-67$.

[15] M. Vaz, R. Solano, Postbuckling analysis of slender elastic rods subjected to uniform thermal loads, Journal of Thermal Stresses 26 (9) (2003) $847-860$.

[16] S. Li, X. Song, Large thermal deflections of Timoshenko beams under transversely non-uniform temperature rise, Mechanics Research Communications 33 (1) (2006) $84-92$.

[17] S. Li, Y. Zhou, Geometrically nonlinear analysis of Timoshenko beams under thermomechanical loadings, Journal of Thermal Stresses 26 (9) (2003) $861-872$.

[18] L. Virgin, Vibration of Axially Loaded Structures, Cambridge University Press, 2007.

[19] R. Plaut, L. Virgin, Vibration and snap-through of bent elastica strips subjected to end rotations, J Appl Mech 76 (4).

[20] Y. Chandra, I. Stanciulescu, T. Eason, M. Spottswood, Numerical pathologies in snap-through simulations, Submitted .

[21] R. L. Taylor, FEAP A Finite Element Analysis Program, Department of Civil and Environmental Engineering, University of California at Berkeley, Berkeley, California, version 8.3 user manual edn., 2011. 
[22] H. Chen, L. Virgin, Finite element analysis of postbuckling dynamics in plates: Part I: An asymptotic approach, International Journal of Solids and Structures 43 (2006) 3983-4007.

[23] Y.-L. Yeh, C.-K. Chen, H.-Y. Lai, Chaotic and bifurcation dynamics of a simply-supported thermo-elastic circular plate with variable thickness in large deflection, Chaos, Solitons and Fractals 15 (5) (2003) 811 - 829.

[24] F. Armero, J. Simo, A new unconditionally stable fractional step method for non-linear coupled thermomechanical problems, International Journal for Numerical Methods in Engineering 35 (4) (1992) 737 - 766.

[25] J. Simo, C. Miehe, Associative coupled thermoplasticity at finite strains: Formulation, numerical analysis and implementation, Computer Methods in Applied Mechanics and Engineering 98 (1) (1992) 41 - 104, URL http://dx.doi .org/10.1016/0045-7825(92)90170-0.

[26] T. Mitchell, A lower bound on snap-through instability in thermomechanically coupled curved beams, Master thesis, University of Illinois at Urbana-Champaign, 2009.

[27] W. Poon, C. Ng, Y. Lee, Dynamic stability of a curved beam under sinusoidal loading, Proceedings of the Institution of Mechanical Engineers, Part G: Journal of Aerospace Engineering 216 (4) (2002) 209 - 217.

[28] H. Chen, L. Virgin, Finite element analysis of postbuckling dynamics in plates: Part II: A nonstationary analysis, International Journal of Solids and Structures 43 (2006) 4008-4027. 
[29] B. Moghaddasie, I. Stanciulescu, M. Rezaiee-Pajand, The Effect of Imperfections on Critical Load in Elastic Structures, Under review .

[30] J.-S. Park, J.-H. Kim, Suppression of aero-thermal large deflections and snap-through behaviors of composite panels using macro fiber composite actuators, Smart Materials and Structures 13 (6) (2004) 1448 - 1459. 